\title{
A Child with 3 Testes: Polyorchism
}

\author{
Naeem Liaqat, ${ }^{\star}$ Ammara Kanwal, Sadia Jamil, Ghulam Khadija, Sidra Ahmad, Kashif Abbas
}

Department of Pediatric Surgery, Rawalpindi Medical University, Rawalpindi, Pakistan

\author{
Correspondence*: Naeem Liaqat, Department of Pediatric Surgery, Rawalpindi Medical University, Rawalpindi, Pakistan \\ E-mail: simsonian.chaudary@gmail.com \\ (C) 2018, Liaqat et al \\ Submitted: 11-01-2018 \\ Accepted: 23-03-2018 \\ Conflict of Interest: None \\ Source of Support: Nil
}

This is an open-access article distributed under the terms of the Creative Commons Attribution License, which permits unrestricted use, distribution, and reproduction in any medium provided the original work is properly cited.

\section{DEAR SIR}

Polyorchism or supernumerary testicle is a rare congenital anomaly, marked by the presence of more than two Testicles. Triorchidism is so far, the most commonly reported variety.[1] These supernumerary testicles may be intra-scrotal and/or associated with cryptorchidism. Polyorchidism is generally an asymptomatic condition but can be found incidentally due to its association with inguinal hernia, undescended testicles, testicular torsion, and to a lesser extent, to hydrocele.[2]

A 12-year-old boy presented to us, in the outpatient department, with left-sided inguinoscrotal swelling. Testes were palpable in scrotal sac bilaterally. On examination, the swelling was reducible, and silk-glove sign was positive ipsilaterally. The size of testis on both sides was comparable and normal. The diagnosis of left-sided inguinal hernia was made and the patient was booked. During herniotomy, a testis of $1.5 \times 1 \mathrm{~cm}$ was found in the sac, along with a normally descended testis in ipsilateral hemiscrotum having similar size [Figure 1]. On further exploration, this extra testis had no epididymis and vas deferens. Also, the blood supply of this testis was originating from the vessels of the originally descended testis. Since this supernumerary testis had no vas deferens, it was excised and high ligation herniotomy was done. As the originally descended testis was of normal size, so no further biopsy was taken. Post-operative recovery of the patient remained une- ventful, and the patient is doing well to date, in a onemonth follow-up.

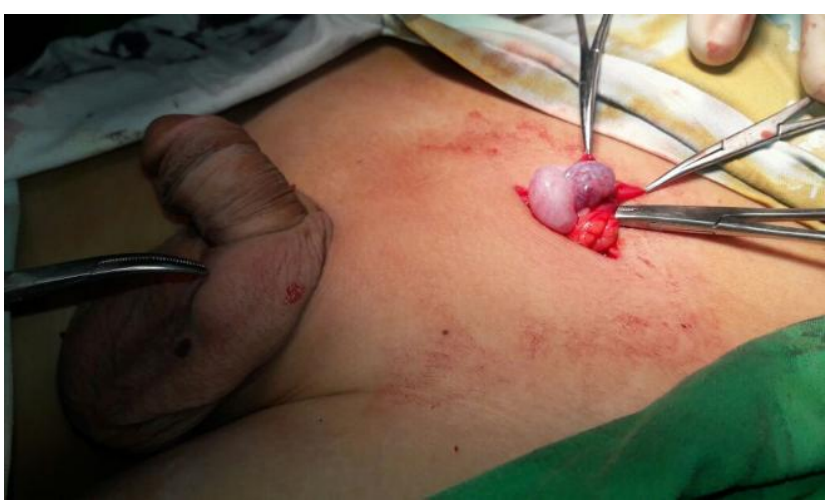

Figure 1: Supernumerary testis. Note that normal testes can be appreciated in the scrotum.

Polyorchidism is a congenital anomaly secondary to abnormal differentiation of genital ridge during embryonic life. Peritoneal bands are considered the culpable agent.[3] Leung classified supernumerary testicles into four types. The extra testicle having no drainage to epididymis or vas deferens was labeled as type I, and the one with its own separate epididymis and vas deferens was categorized as type IV. Type II shares an epididymis and vas deferens with usual ipsilateral testicle whereas type III shares vas deferens only, while having its own epididymis.[2] Our case was of type 1 variety, 
as it had no vas deferens, so it did not have any reproductive potential.

Around 200 cases of polyorchidism have been reported in literature and left-sided extra testicles appear common; triorchidism is common while only 7 cases of 'four testicles' have been reported so far.[4,5] As per anatomical location, intra scrotal variety is most prevalent, followed by inguinal and sometimes intra-abdominal testicles. Commonly they are associated with indirect inguinal hernia. Around $15 \%$ of these cases present with testicular torsion and only $16 \%$ of the cases reported as asymptomatic.[6] If suspected, supernumerary testicles should be confirmed by an ultrasound or MRI. Like undescended testes, non-functional extra testicles may also give rise to a neoplasm. A rate of around $6 \%$ has been reported in the literature.[1] Therefore, surgical excision of these extra testicles is considered a safe approach and was opted in our case as well.

Nevertheless, the fertility of the patient is a major concern that needs consideration, while making a decision regarding management of polyorchidism. Considering the common occurrence of infertility in polyorchids, it's safe to say that preservation of extra testicle may improve fertility.[6] Hence, surgical excision, or conserving the viable testis, the decision regarding management should be made by patient or guardians. And it should be based on suspected fertility status, as well as the possibility of close observation for any malignant transformation.

\section{REFERENCES}

1. Bergholz R, Wenke K. Polyorchidism: a meta-analysis. J Urol. 2009; 182:2422-7.

2. Tonape T, Singh G, Koushik P, Tumepalli T. Triorchidism: a rare genitourinary abnormality. J Surg Tech Case Rep. 2012; 4:126-8.

3. Balasar M, Sonmez MG, Oltulu P, Kandemir A, Kilic M, Goger YE, et al. Polyorchidism; unilateral, one atrophic undescended double testicles. Urol Ann. 2017; 9:208-10.

4. Bhandarwar AH, Gandhi SS, Patel CB, Wagh AN, Gawli V, Jain NA. Triorchidism: presenting as undescended testis in a case of indirect inguinal hernia. Clinics Practice. 2016; $6: 786$.

5. Duymuş M, Menzilcioğlu MS, Çetinçakmak M, Avcu S. A rare case of polyorchidism: four testes. Polish $\mathrm{J}$ Radiol. 2016; 81:39-41.

6. Otero J, Ben-Yakar N. A unique case of intraabdominal polyorchidism: A case study. Case Rep Urol; 2016:2729614. 